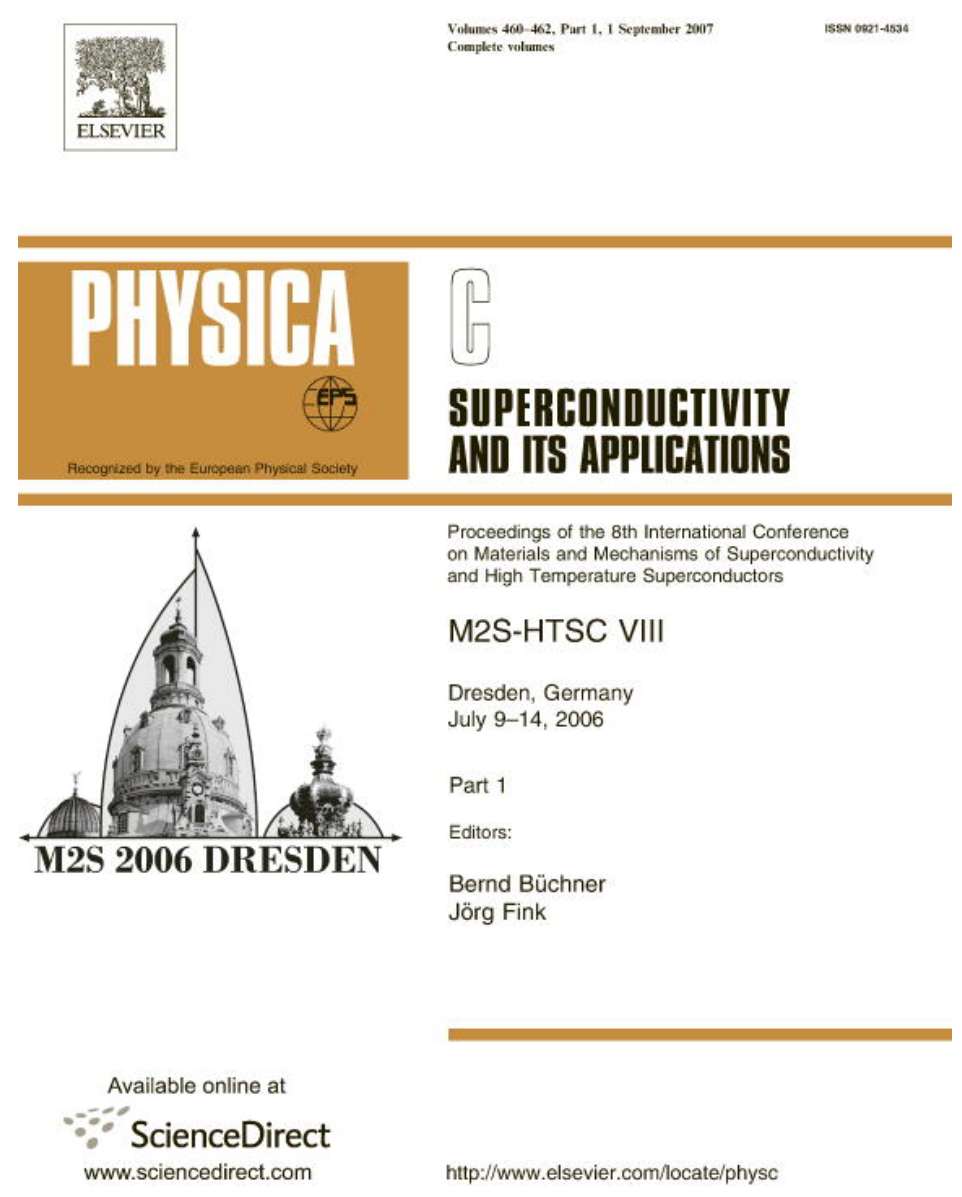

This article was published in an Elsevier journal. The attached copy

is furnished to the author for non-commercial research and education use, including for instruction at the author's institution, sharing with colleagues and providing to institution administration.

Other uses, including reproduction and distribution, or selling or licensing copies, or posting to personal, institutional or third party websites are prohibited.

In most cases authors are permitted to post their version of the article (e.g. in Word or Tex form) to their personal website or institutional repository. Authors requiring further information regarding Elsevier's archiving and manuscript policies are encouraged to visit: 


\title{
Evolution of spin excitations in electron-doped $\operatorname{Pr}_{0.88} \mathrm{LaCe}_{0.12} \mathrm{CuO}_{4-\delta}$
}

\author{
Pengcheng Dai ${ }^{\text {a,b,*, }}$, Stephen D. Wilson ${ }^{\text {a }}$, Shiliang $\mathrm{Li}^{\text {a }}$ \\ a Department of Physics and Astronomy, The University of Tennessee, Knoxville, TN 37996-1200, USA \\ ${ }^{\mathrm{b}}$ Center for Neutron Scattering, Oak Ridge National Laboratory, Oak Ridge, TN 37831-6393, USA
}

Available online 27 March 2007

\begin{abstract}
We briefly review results of recent neutron scattering experiments designed to probe the evolution of antiferromagnetic (AF) order and spin dynamics in the electron-doped $\operatorname{Pr}_{0.88} \mathrm{LaCe}_{0.12} \mathrm{CuO}_{4-\delta}$ as the system is tuned from its as-grown nonsuperconducting AF state into an optimally doped superconductor $\left(T_{\mathrm{c}}=24 \mathrm{~K}\right)$ without static AF order. For under doped materials, a quasi-two-dimensional spindensity-wave was found to coexist with three-dimensional AF order and superconductivity. In addition, the low energy spin excitations follow Bose statistics. In the case of optimally doped material, we have discovered a magnetic resonance intimately related to superconductivity analogous to the resonance in hole-doped materials. On the other hand, the low energy spin excitations have very weak temperature dependence and do not follow Bose statistics, in sharp contrast to the as-grown nonsuperconducting materials.
\end{abstract}

(C) 2007 Elsevier B.V. All rights reserved.

Keywords: Electron-doped high- $T_{\mathrm{c}}$ superconductors; Magnetic excitations

\section{Introduction}

The high-transition-temperature superconductors are fundamentally composed of two-dimensional (2D) copper oxygen planes into which charge carriers, either holes or electrons are doped. Much effort in neutron scattering studies of superconducting copper oxides over the past twenty years has been devoted to hole-doped materials such as $\mathrm{La}_{2-x} \mathrm{Sr}_{x} \mathrm{CuO}_{4}$ (LSCO) and $\mathrm{YBa}_{2} \mathrm{Cu}_{3} \mathrm{O}_{6+x}$ (YBCO) $[1,2]$. A unifying picture of the magnetic excitations have now been achieved for these two mostly studied copper oxide materials $[3,4]$. At low energies, magnetic fluctuations are split away from the wave vector $\mathbf{Q}=(1 / 2,1 / 2)$ in the two-dimensional reciprocal space of the $\mathrm{CuO}_{2}$ planes, which corresponds to the antiferromagnetic (AF) Bragg position of the undoped compounds (Fig. 1a). With increasing energy, the incommensurate spin fluctuations

\footnotetext{
* Corresponding author. Address: Department of Physics and Astronomy, The University of Tennessee, Knoxville, TN 37996-1200, USA. Tel.: +1 865974 1509; fax: +1 8659747843 .

E-mail address: daip@ornl.gov (P. Dai).
}

disperse inward until they reach the commensurate wave vector $\mathbf{Q}=(1 / 2,1 / 2)$, where upon further increases in energy transfer they then disperse back outward rotated from their original orientation. In YBCO, at energies where these incommensurate fluctuations disperse into a commensurate position, a significant enhancement in the spectral weight occurs below $T_{\mathrm{c}}$, and this is known as the resonance mode excitation. The intensity of the resonance is strongly coupled to superconductivity and its energy scales approximately with $k_{\mathrm{B}} T_{\mathrm{c}}$ [5-8].

Although much is known about magnetic excitations in hole-doped materials, relatively little has been done in electron-doped copper oxides because the difficulty in growing large high-quality single crystals required for inelastic neutron scattering experiments. For prototypical electrondoped $\mathrm{Nd}_{2-x} \mathrm{Ce}_{x} \mathrm{CuO}_{4}$ (NCCO) [9], previous neutron scattering experiments show a drastic suppression of the static three-dimensional (3D) AF order when superconductivity is established [10]. However, the static AF order persists even for NCCO with optimal bulk superconductivity $\left(T_{\mathrm{c}}=25 \mathrm{~K}\right)$ [10]. Furthermore, Nd in NCCO has a magnetic ground state, which complicates the interpretation of $\mathrm{Cu}$ magnetism from NCCO [11-13]. For these reasons, 
a
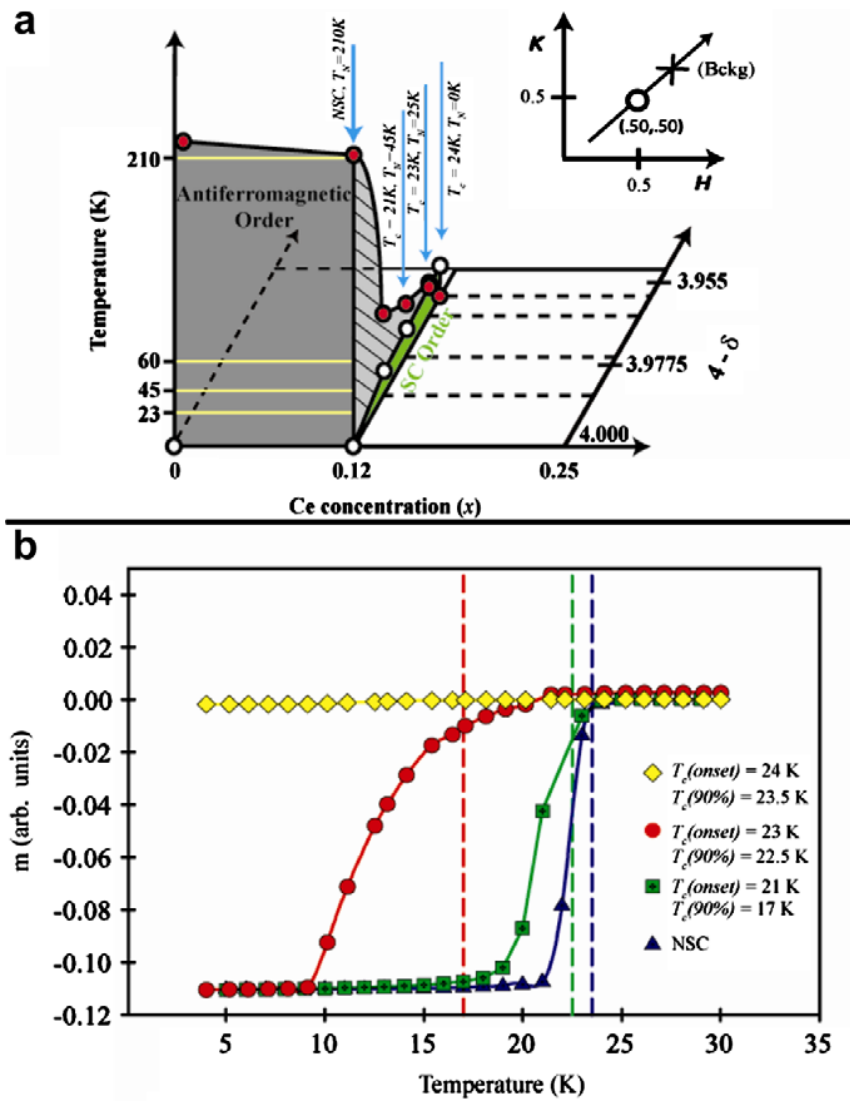

Fig. 1. Phase diagram for single layer electron- and hole-doped copper oxides PLCCO and magnetic susceptibility for PLCCO samples studies. (a) The inset shows the direction of $\mathbf{Q}$-scans with the circle denoting (1/ $2,1 / 2,0$ ) and the cross indicating where the offset background was measured. (b) Bulk susceptibility measurements showing the superconducting transitions for the PLCCO samples studies.

we have focused on another electron-doped material $\mathrm{Pr}_{0.88} \mathrm{LaCe}_{0.12} \mathrm{CuO}_{4}$ (PLCCO), where $\mathrm{Pr}^{3+}$ has a singlet nonmagnetic ground state [14] and the optimally doped concentration is phase pure without static AF order to coexist with superconductivity [15-17]. In the following, we will discuss how spin excitations evolve as PLCCO is annealed from a nonsuperconducting (NSC) antiferromagnet to an optimally doped superconductor with $T_{\mathrm{c}}=$ $24 \mathrm{~K}$.

\section{Neutron scattering results on PLCCO}

Compared to hole-doped materials, one of the unique features of electron-doped copper oxides is that as-grown, these materials are not superconducting. To make superconductivity in electron-doped materials requires annealing of the samples in a reduced oxygen atmosphere, which removes a tiny amount oxygen. Although the precise microscopic role of annealing process to the resulting superconductivity is still under debate [17], it is clear that the phase diagram of electron-doped materials is $3 \mathrm{D}$ where $T_{\mathrm{c}}$ can be controlled by either traversing the Ce concentration and/or tuning the oxygen content in the sample. As a first step, we have carried out neutron scattering experiments to establish the phase diagram of PLCCO as a function of annealing process. In our previous publications $[16,17]$, we have shown that an elastic spin-density-wave (SDW) exists in the under doped PLCCO. We now discuss how low energy spin excitations evolve with increasing superconducting transition temperature through annealing. Fig. 1 shows the susceptibility of the four PLCCO samples used in our experiments. For the as-grown sample, we find that spin excitations are completely controlled by Bose statistics as expected for classical spin-wave excitations. Fig. 2 shows Q-scans for energy transfers $E=0.5,1.5$ and $4.0 \mathrm{meV}$ at different temperatures. At $T=1.4 \mathrm{~K}$, spin-wave excitations are gapped at $E=0.5 \mathrm{meV}$ and the intensity of the scattering increases with increasing energy. The Qwidths of the scattering are entirely resolution-limited and can be well-fitted by Gaussians on linear backgrounds. This is consistent with the very large magnetic exchange $(J>100 \mathrm{meV})$ coupling in the as-grown materials [18]. To test whether the temperature dependence of the spin-wave excitations follow Bose statistics, we plot in Fig. 3 the Qscan data as a function of temperature. For energies above the spin-gap, the spin-wave excitations increase in intensity
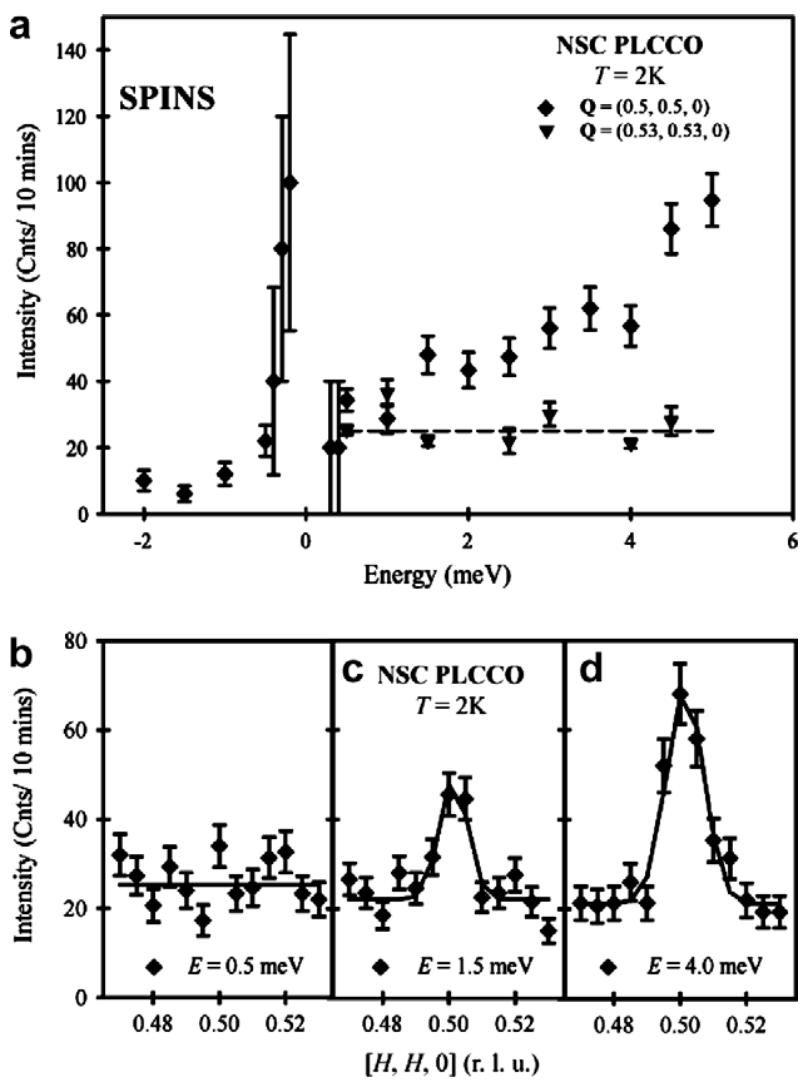

Fig. 2. Low energy excitations in NSC PLCCO. (a) Constant-Q scans at signal $[\mathbf{Q}=(0.5,0.5,0)]$ and background $[\mathbf{Q}=(0.53,0.53,0)]$ positions at $2 \mathrm{~K}$. The dashed line is a linear fit to the energy dependence of the nonmagnetic background. (b)-(d) Constant energy scans along the $[H, H, 0]$ direction at $E=0.5,1.5$, and $4.0 \mathrm{meV}$. Solid lines are Gaussian fits on linear background described in the text. 


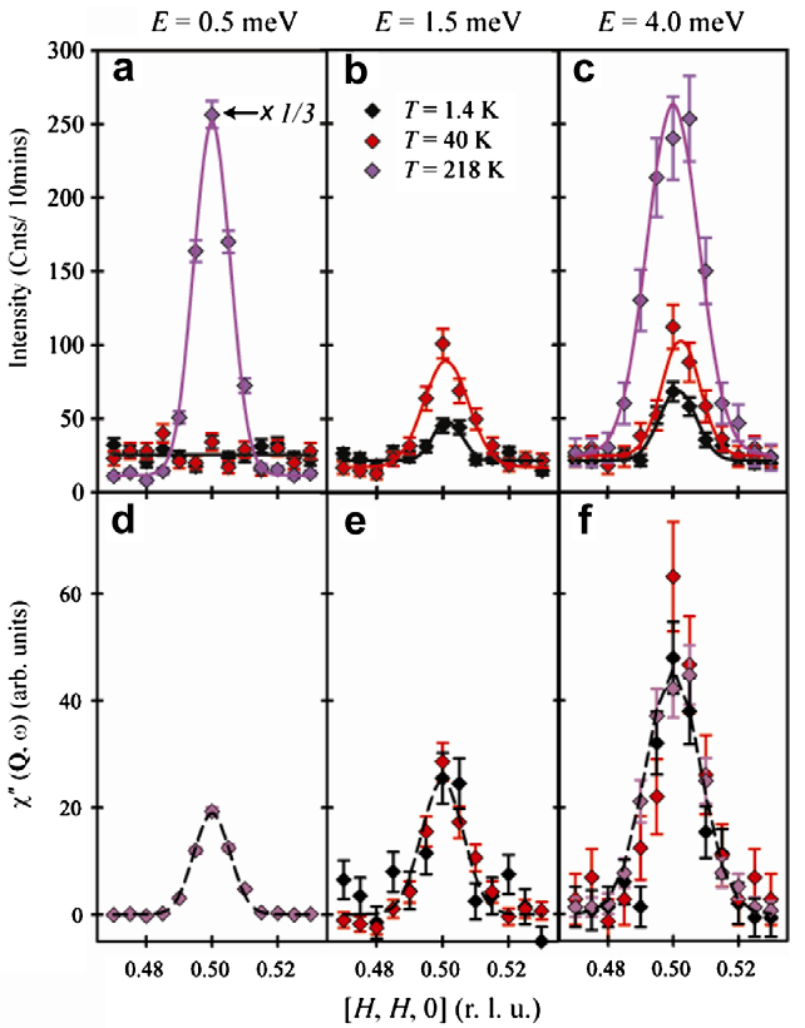

Fig. 3. Temperature dependence of the low energy excitations in NSC PLCCO. (a)-(c) Raw Q-scans around $(0.5,0.5,0)$ at different temperatures at $E=0.5,1.5$, and $4.0 \mathrm{meV}$. A clear un-isotropy spin gap at $0.5 \mathrm{meV}$ persists above $40 \mathrm{~K}$ until the $3 \mathrm{D}$ order breaks down above $T_{\mathrm{N}}$. (d)-(f) Measured dynamic susceptibility at different temperatures for these three energy transfers. Besides the data at $0.5 \mathrm{meV}$, it is clear that susceptibility follows the standard Bose statistics.

with increasing temperature following the Bose population factor. Removing the nonmagnetic background contributions to the scattering, we find that the imaginary part of the dynamic susceptibility, $\chi^{\prime \prime}(\mathbf{Q}, \omega)$, becomes temperature independent (Fig. 3d-f). This is a direct indication that spin-wave excitations in as-grown PLCCO follow Bose statistics.

Now turning to superconducting PLCCO, one can tune $T_{\mathrm{c}}$ by simply annealing the samples at different temperatures. Because of the space limitation, we will only discuss magnetic excitation results for optimally doped PLCCO $\left(T_{\mathrm{c}}=24 \mathrm{~K}\right)$. Fig. 4 shows similar scans as that of Fig. 2 for this material [19]. In remarkable contrast to Fig. 2, the scattering at $E=0.5,1.5$ and $4.0 \mathrm{meV}$ is essentially temperature independent for temperature from $2 \mathrm{~K}$ to $30 \mathrm{~K}$ but decreases slightly at $55 \mathrm{~K}$ owing to the increased background scattering. Compared to the resolution-limited Q-widths in as-grown PLCCO (Fig. 2), the scattering centered around $\mathbf{Q}=(1 / 2,1 / 2)$ is considerably broader. Fourier transforms of the Gaussian peaks give dynamic spin correlation lengths of $\xi \sim 96,80$, and $94 \AA$. The temperature dependence of the scattering at $\mathbf{Q}=(1 / 2,1 / 2)$ confirms the results of the $\mathbf{Q}$-scans. To directly compare the results
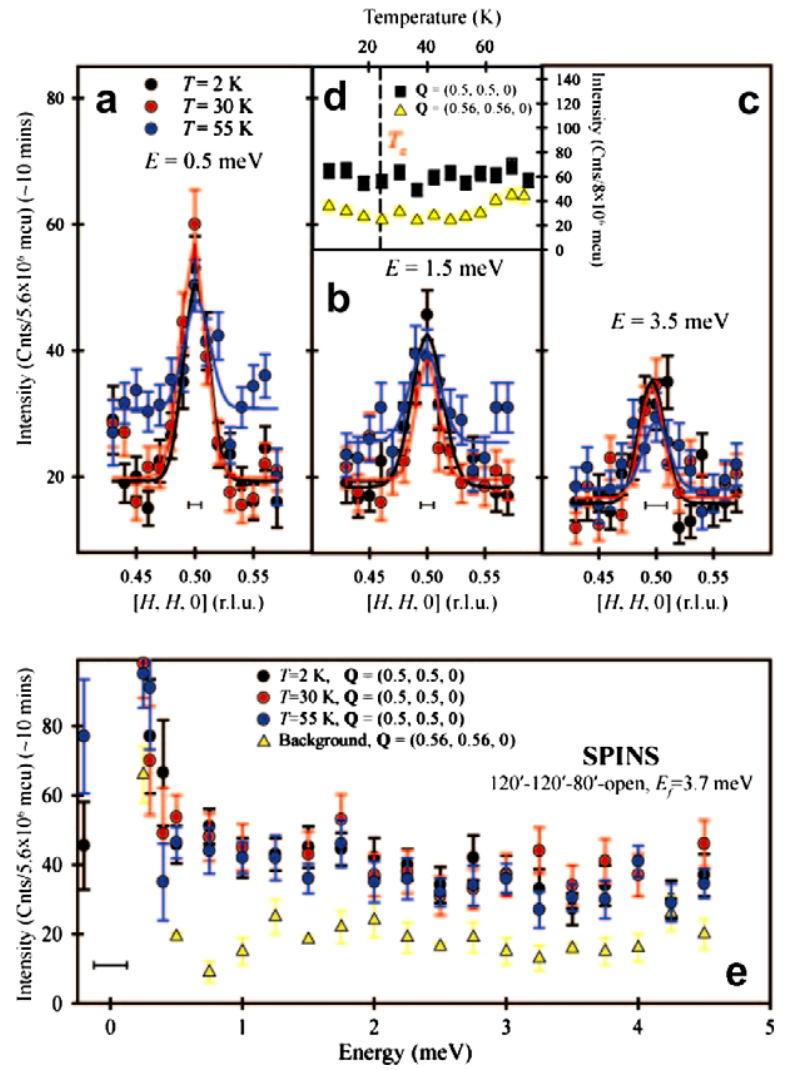

Fig. 4. Temperature dependence of the low energy excitations in superconducting PLCCO using the same spectrometer as Figs. 2 and 3. (a)-(c) Raw Q-scans around $(0.5,0.5,0)$ at different temperatures at $E=0.5,1.5$, and $3.5 \mathrm{meV}$. (d) Temperature-dependent scattering at $Q=(1 / 2,1 / 2,0)$ and $(0.56,0.56,0)$ for $E=1.5 \mathrm{meV}$. The background is independent of temperature below $\sim 50 \mathrm{~K}$ and the magnetic signal becomes weaker at $80 \mathrm{~K}$. (e) Constant-Q scans obtained on SPINS spectrometer with collimations as shown, at the ridge of magnetic scattering at 2, 30 and $55 \mathrm{~K}$, compared with the temperature independent background scattering for temperatures between $2 \mathrm{~K}$ and $30 \mathrm{~K}$.

of the superconducting PLCCO with that of the as-grown material, we plot in Fig. 5 the temperature dependence of the scattering together with the extracted $\chi^{\prime \prime}(\mathbf{Q}, \omega)$. It is immediately clear that $\chi^{\prime \prime}(\mathbf{Q}, \omega)$ behaves quite differently from that of the as-grown sample.

Recently, we have discovered that optimally doped PLCCO has a resonance, analogous to that of the holedoped materials [19]. Similar to hole-doped materials, the resonance in electron-doped PLCCO increases in intensity below $T_{\mathrm{c}}$ and its energy scales with $5.8 k_{\mathrm{B}} T_{\mathrm{c}}$ forming a universal plot for all superconducting copper oxides irrespective of electron or hole doping [19]. However, in contrast to hole-doped materials, magnetic scattering below the resonance in electron-doped PLCCO form commensurate and gapless scattering (Figs. 4 and 5). Therefore the hour-glass shaped dispersion in the magnetic scattering of hole-doped superconducting materials may not be a universal feature of all copper oxides. Instead, the resonance itself appears to be a fundamental property of the superconducting copper oxides. 


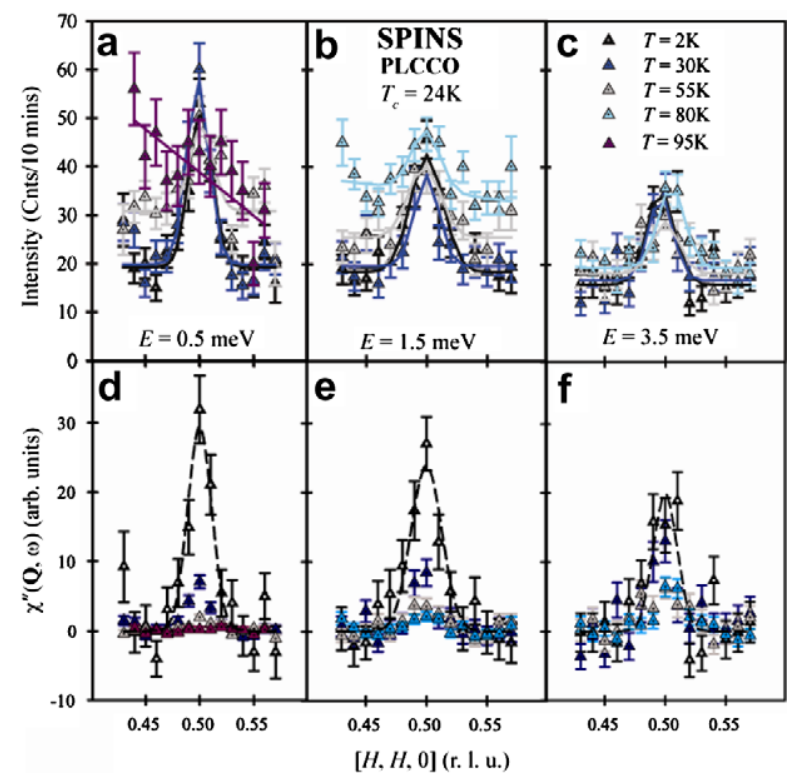

Fig. 5. Temperature dependence of the low energy excitations in superconducting PLCCO. (a)-(c) Raw Q-scans around $(0.5,0.5,0)$ at different temperatures at $E=0.5,1.5$, and $3.5 \mathrm{meV}$. (d)-(f) Temperature dependence of the dynamic susceptibility at these energies are clearly different from those in Fig. 3 for NSC PLCCO.

\section{Conclusions}

The discovery of the resonance in electron-doped PLCCO suggests that resonance may play an essential role in the mechanism of high- $T_{\mathrm{c}}$ superconductivity. While the intensity of the resonance in hole-doped materials appears to arise from opening of a spin-gap in low energy part of the spin excitations spectrum [3-8], the gapless and weakly temperature-dependent low energy magnetic scattering in optimally electron-doped PLCCO is puzzling. At present, it is not clear where the resonance draws its spectral weight below the superconducting transition temperature. On the other hand, the weakly temperature dependent behavior observed in the low-energy spin excitations of PLCCO remarkably resembles the quantum critical scattering seen in heavy fermion compounds [20-22]. To determine the microscopic origin of the resonance and its correlation with high- $T_{\mathrm{c}}$ superconductivity, one must now systematically study the doping, magnetic field, and energy dependence of the mode for different electron-doped materials.

\section{Acknowledgements}

The work reviewed in this paper is performed in collaboration with J.-H. Chung, H.J. Kang, and J.W. Lynn of NIST. We are also grateful to Y. Ando's group for providing single crystals of PLCCO in the early part of our work. This work is supported by the US NSF Grant No. DMR0453805 and also by DOE BES Grant No. DE-FG0205ER46202. ORNL is supported by DOE Grant No. DE-AC05-00OR22725 with UT/Battelle LLC.

\section{References}

[1] M.A. Kastner, R.J. Birgeneau, G. Shirane, Y. Endoh, Rev. Mod. Phys. 70 (1998) 897.

[2] J.M. Tranquada, cond-mat/0512115.

[3] S.M. Hayden, H.A. Mook, Pengcheng Dai, T.G. Perring, F. Dogan, Nature 429 (2004) 531.

[4] J.M. Tranquada et al., Nature 429 (2004) 534.

[5] J. Rossat-Mignod et al., Physica C 185 (1991) 86.

[6] H.A. Mook et al., Phys. Rev. Lett. 70 (1993) 3490.

[7] H.F. Fong et al., Phys. Rev. B 61 (2000) 14773.

[8] Pengcheng Dai, H.A. Mook, R.D. Hunt, F. Dogan, Phys. Rev. B 63 (2001) 054525.

[9] Y. Tokura, H. Takagi, S. Uchida, Nature 337 (1989) 345.

[10] K. Yamada et al., Phys. Rev. Lett. 90 (2003) 137004.

[11] H.J. Kang et al., Nature 423 (2003) 522.

[12] M. Matsuura et al., Phys. Rev. B 68 (2003) 144503.

[13] P.K. Mang et al., Phys. Rev. B 70 (2004) 094507.

[14] A.T. Broothroyd, S.M. Doyle, D. MaK Paul, R. Osborn, Phys. Rev. B 45 (1992) 10075.

[15] M. Fujita et al., Phys. Rev. B 67 (2003) 014514.

[16] Pengcheng Dai et al., Phys. Rev. B 71 (2005) 100502 (R).

[17] H.J. Kang et al., Phys. Rev. B 71 (2005) 214512.

[18] S.D. Wilson et al., Phys. Rev. Lett. 96 (2006) 157001.

[19] S.D. Wilson et al., Nature 442 (2006) 59.

[20] A. Schroder et al., Nature 407 (2000) 351.

[21] C. Aronson et al., Phys. Rev. Lett. 87 (2001) 197205.

[22] S.D. Wilson et al., Phys. Rev. Lett. 94 (2005) 056402. 